\title{
An adaptive ultrasonic backscattered signal processing technique for instantaneous characteristic frequency detection
}

\author{
Bo Jin ${ }^{\mathrm{a}, *}$ and Mang I Vai ${ }^{\mathrm{a}, \mathrm{b}}$ \\ ${ }^{a}$ Biomedical Engineering Laboratory, Department of Electrical and Computer Engineering, Faculty \\ of Science and Technology, University of Macau, Macau SAR, China \\ ${ }^{b}$ State Key Laboratory of Analog and Mixed-Signal VLSI, Department of Electrical and Computer \\ Engineering, Faculty of Science and Technology, University of Macau, Macau SAR, China
}

\begin{abstract}
Ultrasonic diagnosis that is convenient and nondestructive to the human body is widely used in medicine. In clinical, ultrasonic backscattered signals characteristics are utilized to acquire information of the human body tissues to perform diagnosis. In this paper, an adaptive ultrasonic backscattered signal processing technique for instantaneous characteristic frequency detection based on the marginal spectrum is presented. In the beginning, the ultrasonic backscattered signal is decomposed into a series of intrinsic mode functions (IMFs) by the Ensemble Empirical Mode Decomposition (EEMD) algorithm. Then the Hilbert spectrum is gained by the Hilbert transform on the IMFs decomposed and screened. Finally, the timefrequency information in the Hilbert spectrum is utilized to extract the instantaneous characteristic frequency based on the marginal spectrum features to detect the objective. With this technique, the spacing between tissues can be estimated for tissue characterization by processing multiple echoes even in the complicated environment. In the simulation study, comparing with the FFT, the technique presented shows its strong noise immunity and indicates its validity in instantaneous characteristic frequency detection.
\end{abstract}

Keywords: Ultrasonics, EEMD, Hilbert spectral analysis, instantaneous characteristic frequency, simulations

\section{Introduction}

Ultrasonography that is convenient and nondestructive to the human body is widely used in medicine. In clinical, ultrasonic backscattered signals are used to obtain human body tissues characterization to perform diagnosis. L. Landini et al. used spectral analysis on ultrasonic backscattered signals to relate microstructural features with signature of spectrum [1]. J. Girault et al. studied on the ultrasonic backscattered signals attenuation phenomenon by autoregressive analysis to estimate tissue characterization [2]. D. Ta et al. performed processing on ultrasonic backscattered signals to gain the AR cep-

\footnotetext{
${ }^{*}$ Corresponding author: Bo Jin, Biomedical Engineering Laboratory, Department of Electrical and Computer Engineering, Faculty of Science and Technology, University of Macau, Macau SAR, China. Tel.: +853-63763369; Fax: +86-02584631120; E-mail: gfkbsncz@gmail.com.
} 
strum to estimate the mean spacing of trabecular bones [3]. X. Zheng et al. measured corneal thickness based on ultrasonic backscattered signals of before and after surgical operation [4]. T. Liu et al. used 2D spectrum method to analyze ultrasonic backscattered signals for prostate-cancer diagnosis [5]. H. Fang applied ultrasonic backscattered signals in the diagnosis of choroidal hemangioma and choroidal malignant melanoma [6].

In recent years, Joint Time-Frequency Analysis, which can provide signal distribution on both time domain and frequency domain, becomes a hot spot of signal processing. Some classic time-frequency analytic techniques are listed as the Wavelet Transform (WT), the Short-time Fourier Transform (STFT), the Bilinear time-frequency distribution function, the Gabor-Wigner distribution function and the Modified Wigner distribution function. Due to the nonlinear and nonstationary property of ultrasonic backscattered signal, an adaptive time-frequency processing technique is required. The HilbertHuang Transform (HHT) developed by E. Huang et al. [7] is a development adaptive method to extract the features of nonlinear and nonstationary signals, which is widely used in many fields. In addition, the HHT can provide time-frequency distribution graph with relatively high resolution. The advantage of the HHT is obvious comparing with other time-frequency techniques. The Empirical Mode Decomposition (EMD), a core part of the HHT, was applied mainly on noise suppression of ultrasound echoes in the twenty-first century by Y. Mao et al. [8] and Q. Zhang et al. [9]. The Bivariate EMD was applied to perform phase synchrony analysis on ultrasonic echoes signal by M. Leo et al. [10]. However, in practical applications the EMD has many deficiencies one of which is "Mode Mixing" problem caused by intermittency. The Ensemble Empirical Mode Decomposition (EEMD) algorithm developed by $\mathrm{Z}$. Wu et al. possesses the advantages of the EMD and makes up its partial shortcomings [11]. To sum up, the EEMD algorithm is selected to perform analysis on the ultrasonic backscattered signals.

This paper mainly contains four sections. In section 1, an introduction and a brief review of ultrasonic backscattered signals processing are provided. In section 2, the ultrasonic backscattered echoes model is introduced, and the techniques used involving the EEMD and the Hilbert spectral analysis technique are described in theoretical. In section 3, simulation results of noise suppression and instantaneous characteristic frequency detection are presented. In addition, the result comparing with the FFT is presented, and the phenomenon is discussed. In section 4, the conclusion and the future direction are given.

\section{Principle}

In researching process, ultrasonic backscattered echoes model is constructed firstly. Then the EEMD algorithm is performed on the ultrasonic signal simulated. Afterward, IMFs are selected based on maximum power estimation. Finally, the Hilbert transform is implemented on the IMFs selected to perform detection and localization based on the Hilbert spectrum. In this chapter, the concepts related to the processing progress are introduced briefly.

\subsection{Ultrasonic backscattered echoes model [12,13]}

The ultrasonic backscattered echo model is referred as a Gaussian echo model for its envelope of Gaussian-shaped which can be represented as Eq. (1): 


$$
y(t)=\beta e^{-\alpha(t-\tau)^{2}} \cos \left(2 \pi f_{c}(t-\tau)+\phi\right)
$$

In Eq. (1), $\alpha$ indicates the bandwidth factor of the echo, $f_{c}$ indicates the center frequency of the echo, $\tau$ indicates the arrival time of the echo, $\varnothing$ indicates the phase of the signal and $\beta$ indicates the weight of the signal amplitude. For a certain ultrasonic transducer, different test objects will change the values of above parameters.

Therefore, the ultrasonic backscattered echoes can be modeled as Eq. (2) with the form of a superposition of Gaussian echoes each one of which has the form of Eq. (1).

$$
s(t)=\sum_{m=1}^{M} c_{m} e^{-\alpha_{m}\left(t-\lambda_{m}\right)^{2}} \cos \left(2 \pi f_{m}\left(t-\lambda_{m}\right)+\phi_{m}\right)
$$

In Eq. (2), $m$ represents the number of echoes, $\alpha_{m}$ indicates the bandwidth factor corresponding to $m, f_{m}$ indicates the center frequency of the echo corresponding to $m, \lambda_{m}$ indicates the arrival time of the echo corresponding to $m, \emptyset_{m}$ indicates the phase of the signal corresponding to $m$ and $c_{m}$ indicates the weight of the signal amplitude corresponding to $m$. Thus, a series of ultrasonic backscattered echoes are simulated with noise-free by Eq. (2), which is presented in section 3.

\subsection{Ensemble Empirical Mode Decomposition (EEMD)}

The Empirical Mode Decomposition (EMD) algorithm can decompose a signal to a string of Intrinsic Mode Functions (IMFs) by the sifting process [7]. The final result of the EMD indicates input signal consists of a series of IMFs and a residue, which can be represented by Eq. (3):

$$
x(t)=\sum_{i=1}^{n} c_{i}+r_{n}
$$

In Eq. (3), $x(t)$ is a nonlinear and nonstationary input signal, $c_{i}$ represents a IMF corresponding to $i$ and $r_{n}$ represents a residual term.

The Ensemble Empirical Mode Decomposition (EEMD) [11] is a noise-assisted signal processing method which eliminates mode mixing effectively to make the IMFs decomposed possess clear and definite physical meanings. In the EEMD algorithm progress, $\mathrm{N}$ series of the Gaussian white noise with statistical characteristics are added on the nonlinear and nonstationary input signal $\mathrm{N}$ times. Thus, $\mathrm{N}$ series of the input signals with Gaussian white noise are regarded as $\mathrm{N}$ ensembles that are performed by the EMD process separately. The final IMFs are derived by averaging IMFs corresponding to distinct ensembles ultimately, which is represented by Eq. (4):

$$
x(t) \square \frac{1}{N} \sum_{I=1}^{N} E_{I}(t)=\sum_{i=1}^{n} c_{i}+r_{n}
$$


In Eq. (4), $x(t)$ is a nonlinear and nonstationary input signal, $E_{I}(t)$ represents the ensemble corresponding to $I, c_{i}$ represents the averaged IMF corresponding to $i$ and $r_{n}$ represents the averaged residual term. Adding Gaussian white noise process in the EEMD progress can balance the intermittence in the signal to eliminate the mode mixing problem because the power spectrum density of the white noise is uniformly distributed in the frequency domain. Because the mean value of the white noise is zero, the process of averaging the groups of ensembles can eliminate the noise effectively to obtain the original signal. The more ensembles can result in the better results. In summary, the EEMD algorithm is an improvement of the EMD algorithm.

\subsection{Hilbert Spectral Analysis (HSA) [7]}

The Hilbert Transform (HT) is based on the concept of the instantaneous frequency (IF) which is a single-valued function of time, which means that at any time there is only one IF. Thus, the HT emphasizes the local characteristics of input signals.

After achieving the IMFs through above processes, each IMF which makes the IF be meaningful is performed by the Hilbert Transform as Eq. (5) in which $P$ represents the Cauchy principal value:

$$
d_{i}(t)=\frac{1}{\pi} P \int_{-\infty}^{\infty} \frac{c_{i}\left(t^{\prime}\right)}{t-t^{\prime}} d t^{\prime}
$$

The input signal can be expressed as Eq. (6) with instantaneous frequency (IF) after performing the Hilbert transform (HT) on each IMF.

$$
x(t)=\sum_{i=1}^{n} c_{i}+r_{n}=\operatorname{Re}\left(\sum_{i=1}^{n} a_{i}(t) \exp \left(j \int \omega_{i}(t) d t\right)\right)+r_{n}
$$

Thus, the Hilbert spectrum can be defined as Eq. (7) in three-dimensional space of the amplitude $a_{i}(t)$, time $t$ and frequency $\omega_{i}(t)$.

$$
H(\omega, t)=\operatorname{Re}\left(\sum_{i=1}^{n} a_{i}(t) \exp \left(j \int \omega_{i}(t) d t\right)\right)
$$

Furthermore, the marginal spectrum $h(\omega)$ which indicates the contribution of energy on each frequency value in the total time span is defined as Eq. (8):

$$
h(\omega)=\int_{0}^{T} H(\omega, t) d t
$$


Table 1

The parameters of ultrasonic backscattered echo signal simulated with noise-free

\begin{tabular}{llllll}
\hline $\mathrm{m}$ & $C_{m}$ & $f_{m}(\mathrm{MHz})$ & $\lambda_{m}(\mu s)$ & $\alpha_{m}(\mathrm{MHz})^{2}$ & $\emptyset_{m}$ \\
\hline 1 & 5.00 & 8 & 4.5 & 10 & 5 \\
\hline 2 & 4.09 & 8 & 9.0 & 10 & 5 \\
\hline 3 & 3.35 & 8 & 13.5 & 10 & 5 \\
\hline 4 & 2.74 & 8 & 18.0 & 10 & 5 \\
\hline
\end{tabular}

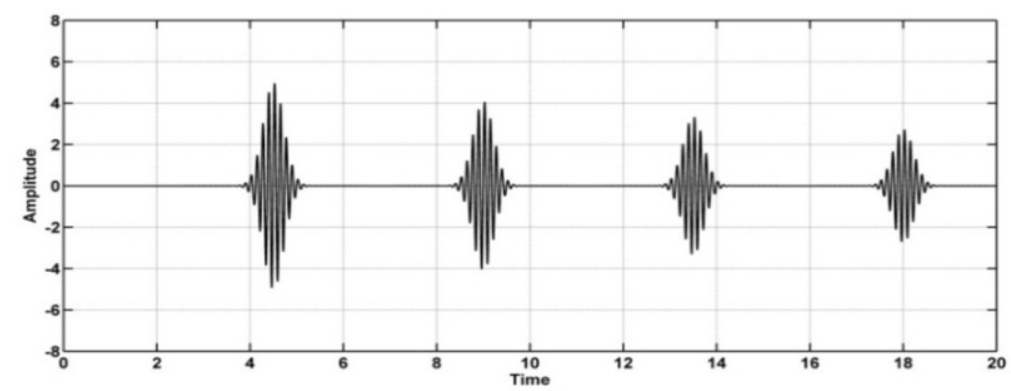

Fig. 1. The signal of ultrasonic backscattered echoes simulated in time domain.

\section{Results and discussion}

In the simulation, the parameters of ultrasonic backscattered signals simulated according to Eq. (2) with noise-free are shown in Table 1.

The characteristic frequency can relate to the location of the different tissue interface. The simulated noise-free typical ultrasonic backscattered signals with amplitude log decrement are shown as Figure 1 with the characteristic frequency of $0.222 \mathrm{MHz}$.

\subsection{A performance investigation on noise suppression}

In this section, the performance of the IMF selection process is investigated by a survey on the noise suppression results. The Gaussian white noise of which the mean value equals zero, the variance equals one is added on the simulated the multiple ultrasonic backscattered echoes so that the signal-tonoise ratio (SNR) of mixed signal equals $-5 \mathrm{~dB}$. The 3D Hilbert time-frequency spectrum of noisy ultrasonic echoes signal ( $\mathrm{SNR}=-5 \mathrm{~dB}$ ) is shown as Figure 2(a). In Figure 2(a), the time-frequency information of ultrasonic backscattered echoes is polluted seriously, and the significant waveform is damaged by noise. The mixed signal is performed by the EEMD with 50 ensembles and 0.2 standard deviation of added noise. Then IMFs decomposed should be selected as Eq. (9) for power estimation according to energy concentration property of the ultrasonic signal:

$$
E_{n}(t)=\left(\operatorname{Max}\left[C_{n}(t)\right]\right)^{2}
$$




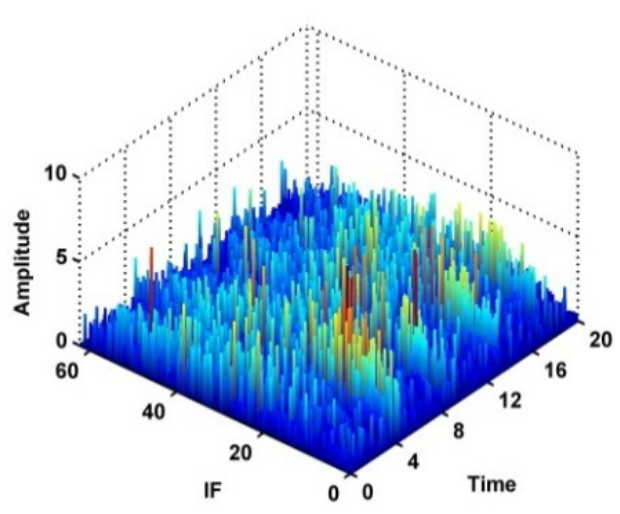

(a)

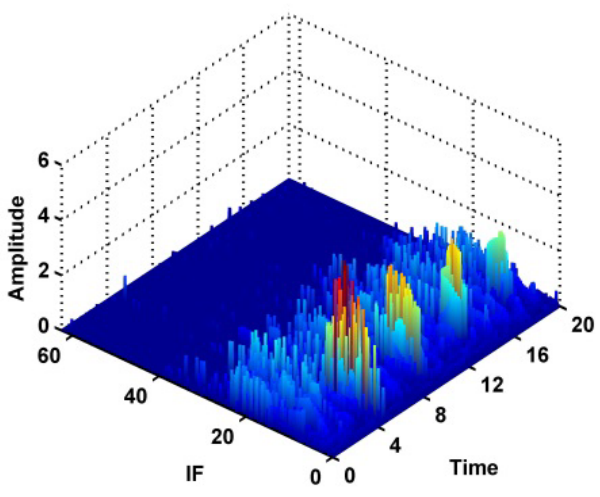

(b)

Fig. 2. The 3D Hilbert TF spectrum.

In the IMF selection process, the final two residue components are deleted for empirical. Then the maximum power estimated of the IMFs achieved after the EEMD process is calculated individually as Eq. (9). Afterwards, the IMFs remained are started from one with maximum power estimated to the final IMF.

The 3D Hilbert time-frequency spectrum of ultrasonic echoes signal by the selected IMFs is shown as Figure 2(b). By IMFs screened reconstruction, the SNR of $2.09 \mathrm{~dB}$ is reached in this case. Through the IMFs selection process, the noise of high frequency section is mostly filtered, and the noise of low frequency section is reduced so that the Hilbert time-frequency spectrum looks more clearly and more concentrate from Figure 2(b) seen. It is more important that the time-frequency information could be restored for characteristic frequency detection and localization.

Furthermore, for proving the effectivity of the IMF selection process, noise suppression performances of the proposed algorithm are investigated by reconstruction of the IMFs screened. The results in three indicators that are the signal to noise ratio (SNR), the mean square error (MSE), and the correlation coefficient (Corr. Coeff.) can be shown as Figure 3. In Figure 3, the SNR and the Corr. Coeff. increase clearly while the MSE (log scale) decreases clearly through the EEMD and IMFs selection process (in the red line).

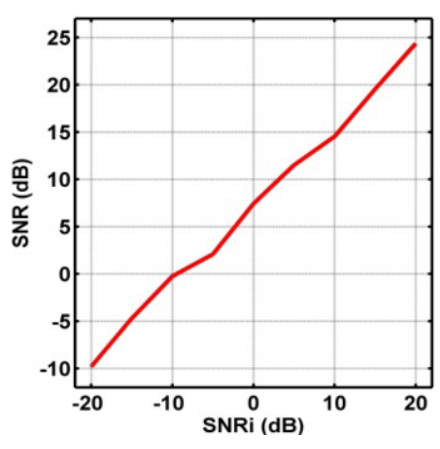

(a)

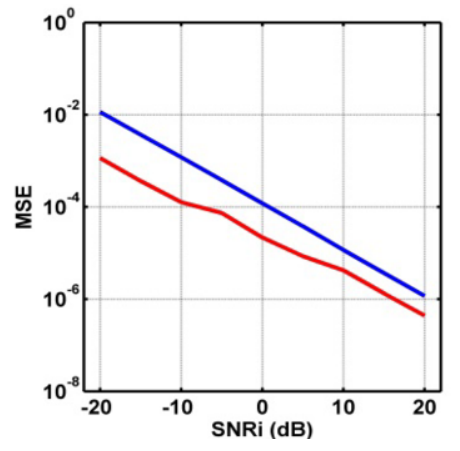

(b)

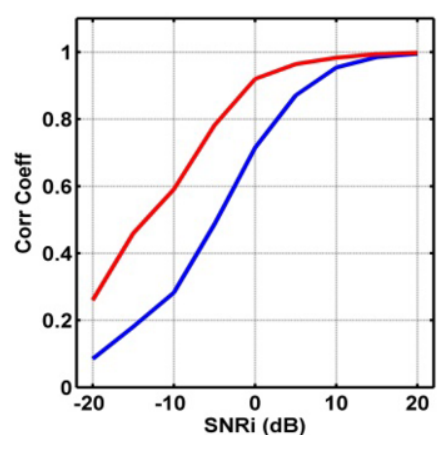

(c)

Fig. 3. Noise suppression performances. 


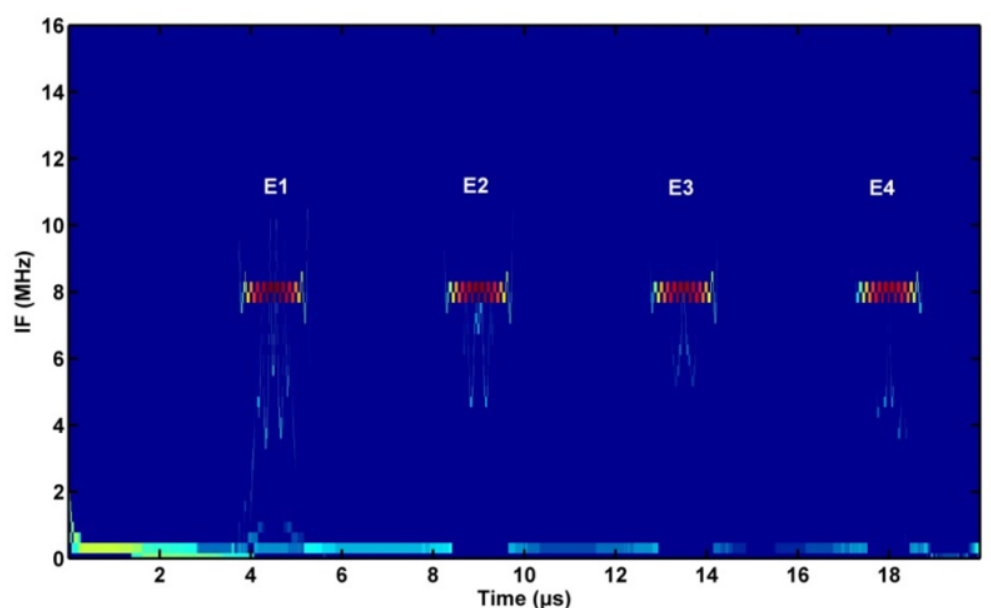

Fig. 4. The 2D Hilbert TF spectrum.

\subsection{Detection and localization}

For noise-free case, the number of ensembles is set as 1 , and the ratio of the standard deviation of the added noise and that of the input signal is set as 0 in the EEMD algorithm. After that, a series of IMFs can be achieved, and the residue term should be removed by convention. Then Hilbert transforms are performed on the IMFs remained as Eq. (7). Thus, the 2D Hilbert Time-frequency can be obtained as Figure 4.

In Figure 4, four groups of echoes can be detected and marked. The characteristic frequency can be estimated by two successive groups. The characteristic frequency lies between $0.105 \mathrm{MHz}$ and $0.285 \mathrm{MHz}$ through transforming time interval from 3.5 to 9.5 microseconds. In addition, some components below $1 \mathrm{MHz}$ in the low frequency section which implies the characteristic frequency information also can be found from Figure 4.

For more detailed information in the frequency domain, the marginal spectrum (see Figure 5) through the transformation of Eq. (8) which indicates the contribution of energy on each instantaneous frequency value can be applied to detect the instantaneous characteristic frequency and perform localization in medicine.

Obviously, the instantaneous characteristic frequency occurs at $0.225 \mathrm{MHz}$ (see Figure 5 (a)) in the low frequency section while the most part of energy concentrates at about $8.023 \mathrm{MHz}$ in the high frequency section, which accords with center frequency of ultrasonic echoes simulated. Comparing with theoretic value, the error of characteristic frequency measured reaches $1.26 \%$. For localization, the location of two tissues interface can be estimated as Eq. (10) where $f_{c}$ indicates the instantaneous characteristic frequency detected and $v$ indicates acoustic speed in medium:

$$
D=\frac{v}{2 f_{c}}
$$




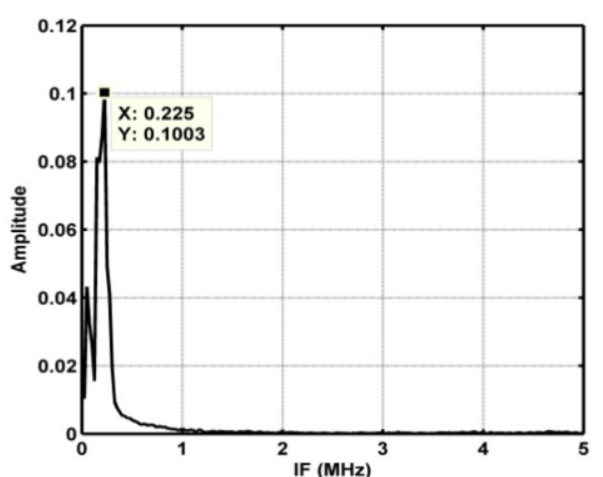

(a)

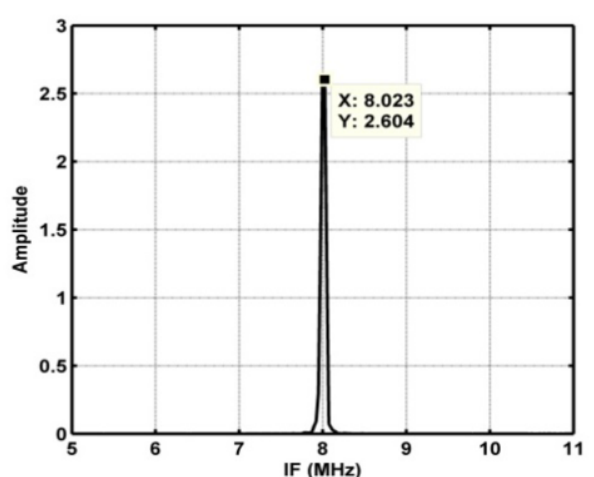

(b)

Fig. 5. The marginal spectrum for noise-free case.

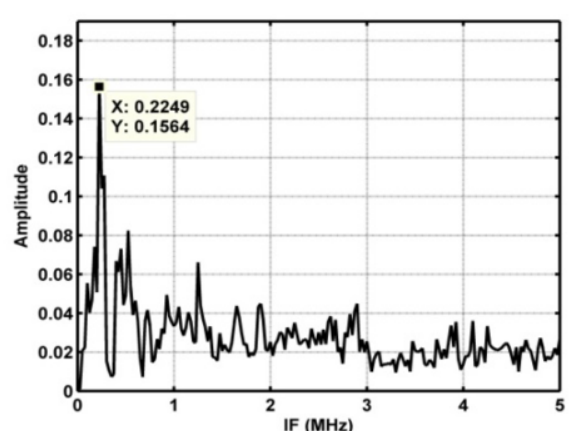

(a)

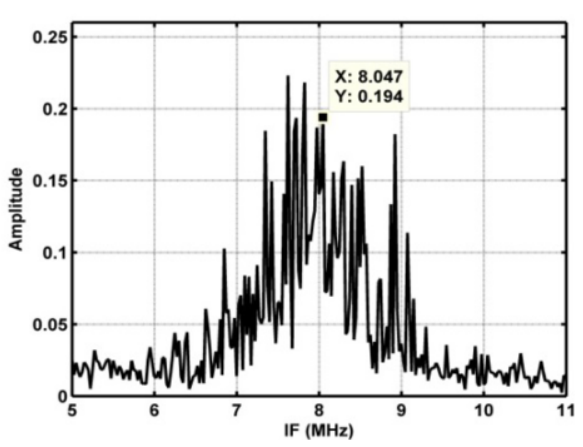

(b)

Fig. 6. Marginal spectrum for noisy case $(\mathrm{SNR}=5 \mathrm{~dB})$.

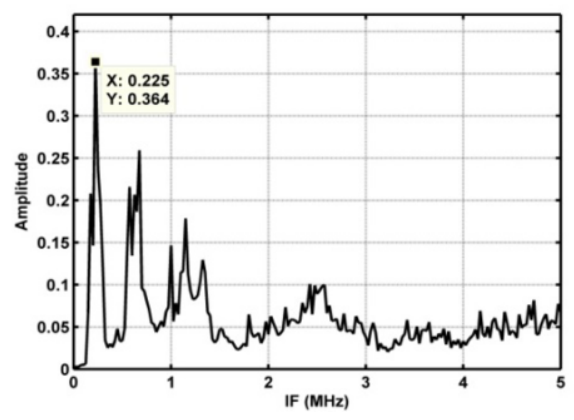

(a)

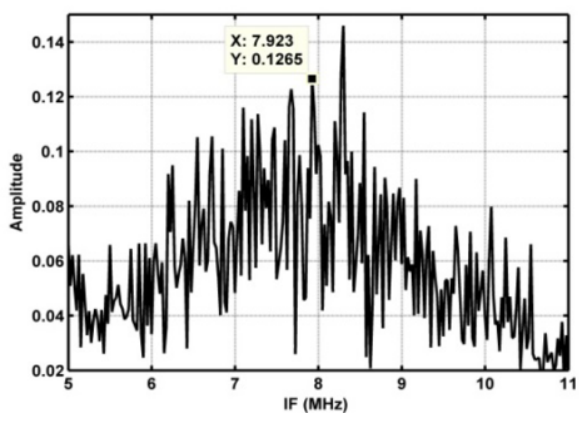

(b)

Fig. 7. Marginal spectrum for noisy case $(\mathrm{SNR}=-5 \mathrm{~dB})$.

For noisy cases, the number of ensembles is set as 50, and the ratio of the standard deviation of the added noise and that of the input signal is set as 0.2 in the EEMD algorithm. After that, the IMF selection process should be applied to remove the unrelated IMFs as described in Chapter 3.1. Hence, Hilbert transforms are performed on the relevant IMFs remained as Eq. (7). For detail information in the frequency domain, the marginal spectrum through the transformation of Eq. (8) can be applied to detect the characteristic frequency and perform localization. The marginal spectrum for the noisy case 
with $\mathrm{SNR}=5 \mathrm{~dB}$ and $\mathrm{SNR}=-5 \mathrm{~dB}$ are indicated as Figures 6 and 7 respectively. In the result, the characteristic frequencies occur obviously at $0.2249 \mathrm{MHz}$ (see Figure $6(\mathrm{a})$ ) and $0.225 \mathrm{MHz}$ (see Figure 7 (a)) in the low frequency section for noisy cases, and harmonic components also appear.

The instantaneous characteristic frequency of the ultrasonic backscattered signals can be extracted in marginal spectrum based on the instantaneous frequency concept through the proposed algorithm from above described. However, the characteristic frequency could not be found in the spectrum by the FFT (in the red line) and the component around the center frequency of $8 \mathrm{MHz}$ are more diffuse than the marginal spectrum (in the blue line) by the proposed algorithm (see Figure 8).

In author's opinion, no matter the frequency concept in the Hilbert spectrum or the marginal spectrum, the frequency type is the instantaneous frequency that is completely different with frequency in the Fourier transform. In the Fourier analysis, the energy in one frequency value represents the existence of a sine wave in the whole time domain. However, the energy represented by an IF value is described as the existence probability of an oscillation wave in it, where a larger Hilbert value means a higher probability. In summarization, the Fourier analysis has limitations on the non-stationary signal since the non-stationary signal is time-varying. Therefore, the reason of that the FFT cannot detect the obvious feature is not difficult to explain.

\section{Conclusion}

In this paper, an adaptive ultrasonic backscattered signal processing technique for instantaneous characteristic frequency detection based on the marginal spectrum is presented. In addition, noiseimmunity of proposed method is investigated and analyzed. In the simulation study, instantaneous characteristic frequency can be detected obviously under noisy environments. Therefore, spacing estimation can be performed for tissue characterization by using the instantaneous characteristic frequency detected in clinical medicine. Comparing with the FFT, the algorithm based on instantaneous frequency proposed is an effective technique. The proposed technique is fit for processing multiple ultrasonic backscattered echoes in a macroscopic view. When more ultrasonic backscattered echoes are obtained, the processing results will be more accurate. In future work, the clinical data will be applied to validate the algorithm proposed.

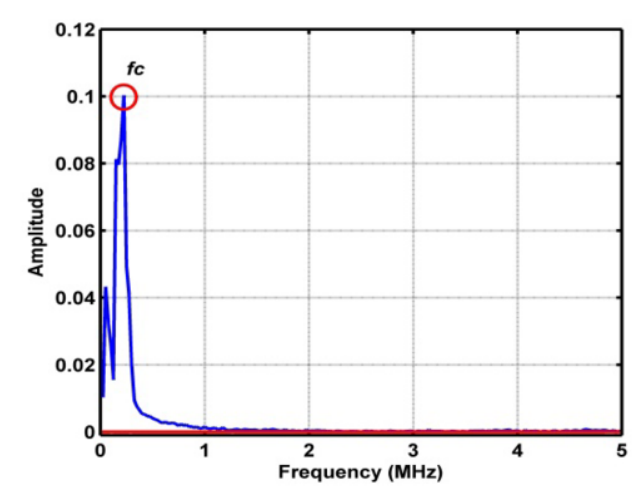

(a)

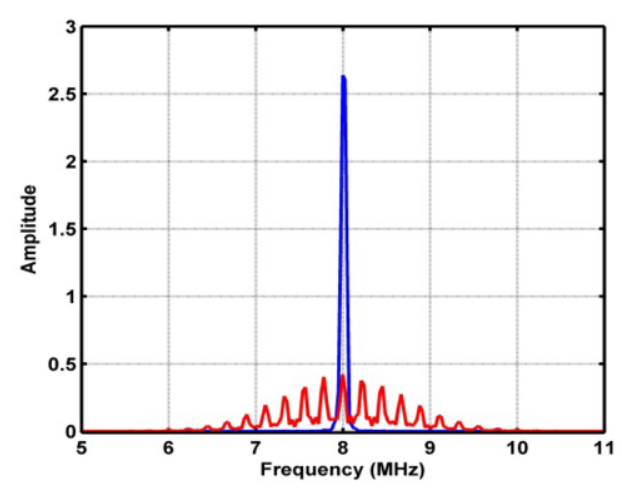

(b)

Fig. 8. Comparison with spectrum by the FFT (Noise-free). 


\section{Acknowledgement}

The authors would like to thank all members of the Biomedical Engineering Laboratory of the University of Macau for their suggestions and technical support. This work is supported by the Research Committee of the University of Macau under Grants RG069/07-08S/MPU/FST \& MYRG076 (Y1-L2)-FST12-MPU.

\section{References}

[1] L. Landini and L. Verrazzani, Spectral characterization of tissues microstructure by ultrasounds: A stochastic approach, IEEE Transactions on Ultrasonics, Ferroelectrics and Frequency Control 37 (1990), 445-456.

[2] J. Girault, F. Ossant, A. Ouahabi, D. Kouamé and F. Patat, Time-varying autoregressive spectral estimation for ultrasound attenuation in tissue characterization, IEEE Transactions on Ultrasonics, Ferroelectrics and Frequency Control 45 (1998), 650-659.

[3] D. Ta, W. Wang, Y. Wang and J. Yu, Estimation of the mean spacing of trabecular bones using autoregression cepstrum, Chinese Journal of Scientific Instrument 28 (2007), 17-22.

[4] X. Zheng, G. Dang, Y. Lei, Y. Su and X. Meng, Measurements of corneal thickness with the Orbscan II Topography System and with A-ultrasonic pachymeter, Journal of Clinical Ophthalmology 16 (2008), 302-304.

[5] T. Liu, M. Mansukhani, M. Benson, R. Ennis, E. Yoshida, P. Schiff, P. Zhang, J. Zhou and G. Kutcher, A feasibility study of novel ultrasonic tissue characterization for prostate-cancer diagnosis: 2D spectrum analysis of in vivo data with histology as gold standard, Medical Physics 36 (2009), 3504-3511.

[6] H. Fang, Combination standard A-scan and high resolution B-scan in the diagnosis of choroidal hemangioma and choroidal malignant melanoma, Chinese Journal of Optometry \& Ophthalmology 5 (2003), 181-183.

[7] E. Huang, Z. Shen, R. Long, Q. Zheng, N. Yen, C. Tung and H. Liu, The empirical mode decomposition and Hilbert spectrum for nonlinear and nonstationary time series analysis, Proceedings of the Royal Society A 454 (1998), 903-995.

[8] Y. Mao and P. Que, Noise suppression and flaw detection of ultrasonic signals via empirical mode decomposition, Russian Journal of Nondestructive Testing 43 (2007), 196-203.

[9] Q. Zhang and P. Que, Improved ultrasonic offshore oil pipeline thickness accurate detection using Hilbert-Huang transform and Elman neural network, Journal of the Japan Petroleum Institute 49 (2006), 321-325.

[10] M. Leo, D. Looney, T. D’Orazio and D. Mandic, Identification of Defective Areas in Composite Materials by bivariate EMD analysis of ultrasound, IEEE Transactions on Instrumentation and Measurement 61 (2012), 221-232.

[11] Z. Wu and E. Huang, Ensemble empirical mode decomposition: A noise-assisted data analysis method, Advances in Adaptive Data Analysis 1 (2005), 1-41.

[12] R. Demirli and J. Saniie, Model-based estimation of ultrasonic echoes part I: Analysis and algorithms, IEEE Transactions on Ultrasonics, Ferroelectrics and Frequency Control 48 (2001), 787-802.

[13] R. Demirli and J. Saniie, Model-based estimation of ultrasonic echoes part II: Nondestructive evaluation applications, IEEE Transactions on Ultrasonics, Ferroelectrics and Frequency Control 48 (2001), 803-811. 